\title{
Repeated High Intensity Bouts with Long Recovery: Are Bicarbonate or Carbohydrate Supplements an Option?
}

\author{
Thomas Stöggl, ${ }^{1,2}$ Rafael Torres-Peralta, ${ }^{3}$ Ebru Cetin, ${ }^{4}$ and Masaru Nagasaki ${ }^{5}$ \\ ${ }^{1}$ Department of Sport Science and Kinesiology, University of Salzburg, Schlossallee 49, 5400 Hallein/Rif, Austria \\ ${ }^{2}$ Swedish Winter Sports Research Centre, Department of Health Sciences, Mid-Sweden University, Studentplan 4, \\ 83140 Östersund, Sweden \\ ${ }^{3}$ Department of Physical Education, University of Las Palmas de Gran Canaria, C/Juan de Quesada, \\ No. 3035001 Las Palmas de Gran Canaria, Spain \\ ${ }^{4}$ Gazi University School of Physical Education and Sports, Gazi University, Teknikokullar, 06500 Ankara, Turkey \\ ${ }^{5}$ Department of Health Science, Faculty of Psychological and Physical Science, Aichi Gakuin University, 12 Araike, \\ Iwasaki-cho, Nisshin, Aichi 470-0915, Japan
}

Correspondence should be addressed to Thomas Stöggl; thomas.stoeggl@sbg.ac.at

Received 28 July 2014; Accepted 25 September 2014; Published 10 November 2014

Academic Editor: David C. Gaze

Copyright (C) 2014 Thomas Stöggl et al. This is an open access article distributed under the Creative Commons Attribution License, which permits unrestricted use, distribution, and reproduction in any medium, provided the original work is properly cited.

The effects of varying recovery modes and the influence of preexercise sodium bicarbonate and carbohydrate ingestion on repeated high intensity performance, acid-base response, and recovery were analyzed in 12 well-trained males. They completed three repeated high intensity running bouts to exhaustion with intervening recovery periods of 25 min under the following conditions: sodium bicarbonate, active recovery (BIC); carbohydrate ingestion, active recovery (CHO); placebo ingestion, active recovery (ACTIVE); placebo ingestion, passive recovery (PASSIVE). Blood lactate (BLa), blood gases, heart rate, and time to exhaustion were collected. The three high intensity bouts had a duration of $138 \pm 9,124 \pm 6$, and $121 \pm 6 \mathrm{~s}$ demonstrating a decrease from bout 1 to bout 3 . Supplementation strategy had no effect on performance in the first bout, even with differences in $\mathrm{pH}$ and bicarbonate $\left(\mathrm{HCO}_{3}{ }^{-}\right)$. Repeated sprint performance was not affected by supplementation strategy when compared to ACTIVE, while PASSIVE resulted in a more pronounced decrease in performance compared with all other interventions. BIC led to greater $\mathrm{BLa}, \mathrm{pH}$, and $\mathrm{HCO}_{3}{ }^{-}$ values compared with all other interventions, while for PASSIVE the opposite was found. BLa recovery was lowest in PASSIVE; recovery in $\mathrm{pH}$, and $\mathrm{HCO}_{3}{ }^{-}$was lower in PASSIVE and higher in BIC.

\section{Background}

Many sports (e.g., team sports such as soccer, rugby, and ice hockey; combat sports like judo; endurance sports like swimming, cycling, and running) often require short duration maximal or near-maximal efforts to be regularly repeated over an extended period of time [1]. In particular, the crosscountry skiing sprint competitions have four maximal bouts with durations of 2-5 minutes within 2-3 hrs (including the qualification bout). In the finals, the skiers have to reproduce maximal performance over 3 bouts with only $10-25$ min rest in between [2]. Therefore, understanding the physiological load and the recovery kinetics of various physiological parameters (e.g., blood pH, blood lactate (BLa), etc.) and their effects on subsequent repeated performance is essential.

During a sprint as short as $30 \mathrm{~s}$, muscle glycogen stores are supposed to be partially consumed and, at the end of long duration training (60-90 min) that involves high, moderate, and low bouts of exercise, muscle glycogen will be dramatically reduced or even depleted [3]. In a simulated alpine skiing slalom training with 16 times $45 \mathrm{~s}$ runs (one run every $20 \mathrm{~min}$ ), it was demonstrated that after the fourth run the intramuscular glycogen store was reduced by $30 \%$ and after 16 runs by $71 \%$, especially in type 1 fibers [4]. Stöggl et al. [2] demonstrated that, during a cross-country skiing sprint, performance and peak BLa decreased from heat to heat 
and the magnitude of BLa was positively related with sprint performance. Based on their results, both Stöggl et al. [2] and Vogt et al. [4] recommended carbohydrate supplementation prior to, during, or after the race/training.

Several studies showed that ergogenic supplements positively affected sprint performance, as well as postperformance recovery (e.g., [5-7]). Carbohydrate (CHO) based supplements are probably the most common. There is an ongoing debate among coaches and athletes concerning whether $\mathrm{CHO}$ supplementation during repeated high intensity sprint competitions is a solution (e.g., among cross-country skiing sprint skiers). However, research on intermittent or high intensity and repeated sprint ability together with $\mathrm{CHO}$ loading or supplementation is sparse $[8,9]$. Supplementation with $\mathrm{CHO}$ prior to or during a time trial resulted in performance improvements due to an increase in $\mathrm{CHO}$ oxidation and maintained plasma glucose levels [10].

Additionally, the use of sodium bicarbonate as one of the major buffering agents used to diminish the supposed negative effects of acidosis is common [11]. Recent research has shown that ingesting sodium bicarbonate may enhance aspects of sprint performance such as power output, total anaerobic work, and delaying fatigue $[11,12]$, though results are not consistent. A recent meta-analysis revealed that ingestion of bicarbonate improves mean power by $1.7 \%( \pm 2.0 \%)$ in high intensity races of short duration [13]. Lavender and Bird [14] and Bishop et al. [15] concluded that sodium bicarbonate supplementation improves power output levels in repeated short duration sprints (e.g., 10 times $10 \mathrm{~s}$ sprints with $50 \mathrm{~s}$ recovery, or 5 times $6 \mathrm{~s}$ all-out sprints every $30 \mathrm{~s}$ ). Bishop et al. [15] suggested that the improved performance was a result of the greater extracellular buffer concentration by sodium bicarbonate ingestion increasing $\mathrm{H}^{+}$efflux from the muscles into the blood and an increased anaerobic energy contribution. Research on repeated high intensity bouts of longer duration and with longer recovery in between is lacking.

Finally, the role of the recovery mode during repeated high intensity activity is also a topic of debate. Active recovery (pedaling at $20 \% \mathrm{VO}_{2 \max }$ ) has been shown to facilitate performance ( 4 times $110 \%$ peak performance to exhaustion with 5 min recovery in between bouts) compared to passive recovery [16], while the opposite was found in intermittent exercise of $15 \mathrm{~s}$ high intensity versus $15 \mathrm{~s}$ recovery (40\% $\mathrm{VO}_{2 \max }$ during active recovery) [17] and with no differences between 3 times $110 \%$ peak power output to exhaustion with 12 min breaks (20\% of maximal workload during active recovery) in between [18]. Furthermore, passive recovery is expected to favor PCr resynthesis during shorter recovery periods between bouts, for example, up to $2 \mathrm{~min}$ [7].

Therefore, the aim of the current study was to analyze the effects of passive versus active recovery and how carbohydrate and sodium bicarbonate supplements influence performance and recovery during three bouts of high intensity exercise with recovery duration of 25 minutes in between. The specific hypotheses were that (a) active recovery facilitates repeated performance, (b) acute sodium bicarbonate ingestion leads to performance enhancement in each high intensity bout, and (c) acute carbohydrate supplementation delays fatigue across all three high intensity bouts.

\section{Methods}

2.1. Subjects. Twelve endurance-trained males (mean $\pm \mathrm{SD}$ : age $=32.8 \pm 3.8$ yrs, body height $=1.78 \pm 0.06 \mathrm{~m}$, body weight $=74 \pm 6 \mathrm{~kg}$, and $\mathrm{VO}_{2 \max }=66.4 \pm 5.2 \mathrm{~mL} \cdot \mathrm{min}^{-1} \cdot \mathrm{kg}^{-1}$ ) with backgrounds in running and cross-country skiing volunteered to participate in this study.

2.2. Ethics Statement. Subjects were informed about the test procedures and possible risks prior to giving their written informed consent to participate. The research techniques and experimental protocol were preapproved by the Local Ethics Committee of the University of Salzburg, and the study was performed in accordance with the Declaration of Helsinki.

2.3. Overall Design of the Study. Participants reported to the laboratory at the same time of the day on four separate test days with a minimum of 5 days, but no more than 8 days between trials. For all participants, all tests were performed within $23 \pm 3$ days. Participants were advised not to perform strenuous exercise within $72 \mathrm{hrs}$ prior to each trial and were asked to control, record, and duplicate food intake 24 hrs prior to each trial (data collected and reviewed, but not presented in this paper). Each test day included a standardized warm-up followed by three all-out sprint bouts running on a treadmill with fixed breaks of $25 \mathrm{~min}$ in between bouts (comparable with the competition mode in sprint cross-country skiing). Each test had a total duration of approximately $90 \mathrm{~min}$. On each test occasion the following supplementation and recovery strategies were randomized (counterbalanced using the Latin least squares design) in a double-blind manner across the participants: (a) placebo drink with no activity between sprint bouts (PASSIVE); (b) placebo drink and low intensity running between sprint bouts (ACTIVE); (c) carbohydrate mix and low intensity running between sprint bouts ( $\mathrm{CHO}$ ); and (d) bicarbonate drink and low intensity running between sprint bouts (BIC).

2.4. Supplementation. The BIC drink was prepared according to McNaughton [19] using $300 \mathrm{mg} / \mathrm{kg}$ (body mass) bicarbonate and $2 \mathrm{cl}$ artificial sweetener (saccharin) dissolved in $6 \mathrm{~mL} / \mathrm{kg}$ (body mass) water. The $\mathrm{CHO}$ mixture was an isotonic $5.5 \%$ solution, which consisted of $1.7 \%$ glucose; $1.1 \%$ fructose; $0.6 \%$ maltose; $1.9 \%$ (higher) saccharide and electrolytes (sodium: $61 \mathrm{mg} / 100 \mathrm{~mL}$ and potassium: $10 \mathrm{mg} / 100 \mathrm{~mL}$ ) [20] with total CHO amount of $300 \mathrm{mg} / \mathrm{kg}$ (body mass). The placebo drink (PASSIVE and ACTIVE group) consisted of artificial sweetener (saccharin) and sodium chloride. For CHO, PASSIVE, and ACTIVE the participants ingested $6 \mathrm{~mL} / \mathrm{kg}$ (body mass) of the respective solution at arrival and the same amount throughout the experiment.

2.5. Experimental Protocol. Participants reported to the laboratory $100 \mathrm{~min}$ before test start with no food intake $3 \mathrm{hrs}$ prior 


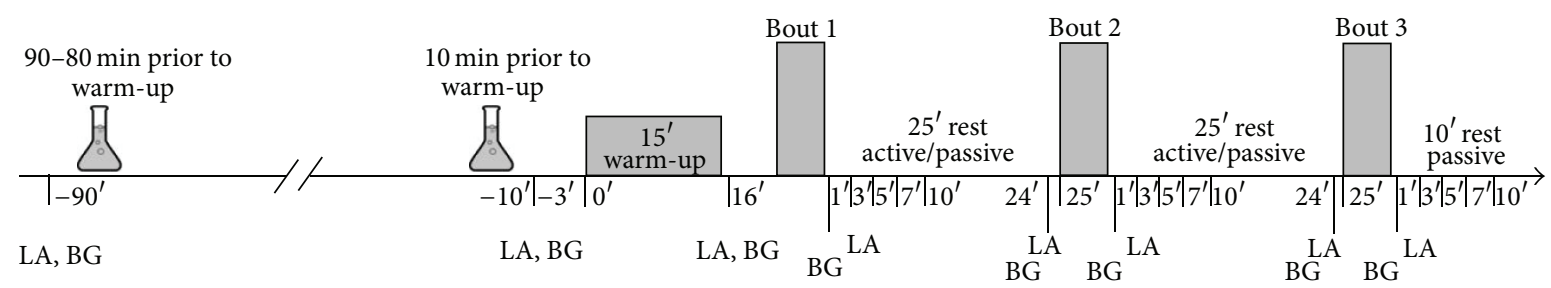

FIGURE 1: Illustration of the general design of the study.

to the test start; they were instructed to be refrained from caffeine and alcohol and be well-hydrated. Ten min after arrival, two blood samples from the earlobe were taken to determine resting values in blood lactate (BLa) and blood gases. Directly afterwards (90 min prior to the start of the exercise protocol) the participants drank, according to the randomized order, one of the three supplements [placebo (PASSIVE, ACTIVE), CHO, and BIC] within a 10 min period. In addition, ten minutes prior to warm-up, as well as within the breaks between the single bouts, participants drank $2 \mathrm{~mL} / \mathrm{kg}$ of CHO or placebo (for PASSIVE, ACTIVE, and BIC). Another two blood samples for BLa and blood gases were collected 3 minutes prior to start of the warm-up. The $\mathrm{CHO}$ was administered throughout the protocol in order to possibly enhance $\mathrm{CHO}$ oxidation and maintain plasma glucose levels [10]. BIC supplementation was switched to the placebo drink during the exercise, in order to prevent possible gastrointestinal problems. The test started with a standardized warm-up protocol of $15 \mathrm{~min}$, with $5 \mathrm{~min}$ at $8 \mathrm{~km} / \mathrm{h}$ at $1.5 \%$ grade, $5 \mathrm{~min}$ at $8 \mathrm{~km} / \mathrm{h}$ at $5 \%$ grade, and 3 times $30 \mathrm{~s}$ acceleration runs up to 16,18 , and $19 \mathrm{~km} / \mathrm{h}$ at $5 \%$ grade with $1 \mathrm{~min}$ at $8 \mathrm{~km} / \mathrm{h}$ in between the sprint runs. The warmup was finished with $1.5 \mathrm{~min}$ at $8 \mathrm{~km} / \mathrm{h}$ at $5 \%$ grade. In the first minute, after warm-up, two blood samples were taken: a blood sample for the determination of BLa and one for the determination of blood gases. After a rest of $3 \mathrm{~min}$, the first high intensity bout started with a fixed treadmill inclination of $5 \%$ and a treadmill speed of $19 \mathrm{~km} / \mathrm{h}$. Participants were asked to run as long as possible at this treadmill speed and were maximally encouraged by the test team. The time to exhaustion was taken as the performance parameter for each sprint bout. The participants had no knowledge of the length of time they were actually running. Even though time trial tests were shown to have higher validity than a time to exhaustion test [21], we decided to use this test concept based on (1) high internal validity by maximal standardization of the running test on an indoor treadmill and (2) prevention of different pacing strategies during each of the four trials. Furthermore, the participants were familiar with this test procedure based on a reliability study prior to this experiment ( $n=15$ including the same subjects of this study, intraclass coefficient of $0.95 ; P<0.001$ for maximal test duration).

After each bout, the participants had a 25 min break consisting of $5 \mathrm{~min}$ rest followed by $17 \mathrm{~min}$ of either sitting on a chair (PASSIVE) or low intensity running [ 65-70\% peak heart rate (HR)] on the treadmill at $6 \mathrm{~km} / \mathrm{h}$ and a $1.5 \%$ grade (ACTIVE, CHO, and BIC) and another 3 min passive rest prior to the second sprint bout. The same procedure was repeated for the third sprint bout. Following each sprint bout blood samples for BLa were taken in the 3rd, 5th, 7th, 10th, and 25th min and for blood gases in the 1st and 24th min. An overview on the entire protocol is illustrated in Figure 1. All participants were secured with a safety harness suspended from the ceiling and attached to an emergency break.

2.6. Instruments. The participants' HR was recorded throughout all tests telemetrically (Suunto t6, Helsinki, Finland) at $2 \mathrm{~s}$ intervals. The peak HR of each bout was used for further statistical analysis. For BLa a $20 \mu \mathrm{L}$ and for blood gases a $150 \mu \mathrm{L}$ blood sample from the hyperaemized right earlobe were collected into a capillary tube (Eppendorf AG, Hamburg, Germany). The BLa samples were immediately analyzed amperometric-enzymatically (Biosen 5140, EKFDiagnostic GmbH, Magdeburg, Germany). The lactate sensor was calibrated before each test and checked using a lactate standard sample of $12 \mathrm{mmol} / \mathrm{L}$. Results within a range of $\pm 0.1 \mathrm{mmol} \cdot \mathrm{L}^{-1}$ were accepted. Blood gases were analyzed using the Cobas b 221 system (Roche Diagnostics GmbH, Mannheim, Germany). The system automatically performs a system calibration every $24 \mathrm{hrs}$ and a 1-p calibration every 30 minutes.

2.7. Statistical Analysis. All data were checked for normality using Shapiro-Wilk's test to exhibit a Gaussian distribution, and the values are presented as mean \pm SD. For determination of global differences between measured physiological parameters (BLa, $\mathrm{pH}, \mathrm{HCO}_{3}{ }^{-}$, base excess (BE), $\mathrm{PO}_{2}$, and $\mathrm{PCO}_{2}$ ) across the entire experiment (all three bouts including the postrecovery values) a $4 \times 5$ repeated-measures ANOVA ( 4 interventions $\times 5$ time points) was applied. For the variables sprint bout time and the peak values of the measured physiological parameters during or after sprint a $4 \times 3$ repeated-measures ANOVA ( 4 interventions $\times 3$ sprint bouts) was conducted. For determination of differences within the single sprint bouts and the single recovery values an ANOVA with repeated measures was used. In all cases a Bonferroni post hoc analysis was applied. Correlations of performance in the first bout to the peak values and recovery values in BLa and $\mathrm{pH}$ Pearson's product moment correlation coefficients were calculated. Level of significance was set at $\alpha<0.05$.

\section{Results}

Upon arrival at the laboratory, there were no differences indicated for $\mathrm{pH}$, lactate, $\mathrm{HCO}_{3}{ }^{-}, \mathrm{BE}, \mathrm{PO}_{2}$, or $\mathrm{PCO}_{2}$ between the 
four interventions (Table 1). Some participants did report experiencing minor bloating and/or gastrointestinal stress following the $\mathrm{BIC}(n=2)$ and $\mathrm{CHO}(n=1)$ within the $90 \mathrm{~min}$ after ingestion time period. However, these side effects subsided by the time the warm-up started.

High intensity running performance reflected by the sprint bout duration demonstrated a main effect for intervention (mean of all three bouts) (PASSIVE: $122 \pm 6 \mathrm{~s}$; ACTIVE: $125 \pm 5 \mathrm{~s}$; CHO: $132 \pm 8 \mathrm{~s}$; BIC: $132 \pm 9 \mathrm{~s} ; P=0.031)$ with no difference at bout 1 , but lower performance in the PASSIVE group at bout 2 compared with BIC $(P=0.048)$ and at bout 3 compared with all other groups $(P=0.009$ to 0.012 ). This was also reflected by an interaction effect towards greater decreases in running performance with PASSIVE compared with BIC from bout 1 to bout $2(P=0.05)$ and when compared with $\mathrm{BIC}$ and $\mathrm{CHO}$ from bout 1 to bout 3 (PASSIVE: $-30.5 \pm 17.9$ s; CHO: $-14.5 \pm 13.2 \mathrm{~s}$; BIC $-9.2 \pm$ 16.9 s; $P=0.029)$. There was a main effect for factor time $(P=0.008)$ demonstrating a tendency towards a decrease in performance $(-10 \%)$ from bout 1 to bout $2(138 \pm 9 \mathrm{~s}$ versus $124 \pm 6 \mathrm{~s}, P=0.066)$ and when comparing bout 1 with bout $3(-12 \%$ : $138 \pm 9 \mathrm{~s}$ versus $121 \pm 6 \mathrm{~s}, P=0.007)$ (Figure 2(a)).

Peak HR within each bout demonstrated a main effect for intervention (PASSIVE: $181 \pm 2 \mathrm{bpm}$; ACTIVE: $185 \pm 2 \mathrm{bpm}$; CHO: $184 \pm 2$ bpm; BIC: $186 \pm 2$ bpm; $P=0.009)$ with higher values in BIC compared with PASSIVE $(P=0.026)$ and a tendency towards higher values in ACTIVE compared with PASSIVE $(P=0.062)$. When analyzing each bout separately, there was no difference between interventions at bout 1 , while at both bout $2(P=0.015)$ and bout $3(P<0.001)$ PASSIVE was lower in peak heart rate compared with all other interventions. A main effect was also found for factor time with a reduction in peak HR across bouts (bout 1: $189 \pm$ $2 \mathrm{bpm}$; bout 2: $182 \pm 2 \mathrm{bpm}$; bout 3: $181 \pm 2 \mathrm{bpm} ; P<$ $0.001)$. An interaction of time $\times$ intervention was found $(P<0.001)$, with greater decreases from bout 1 to bout 2 and bout 1 to bout 3 in PASSIVE compared with all other interventions.

3.1. Peak Values after Sprint Bout. Physiological parameters across the entire experiment for all four interventions are illustrated in Figure 2 and Table 1. Peak BLa values demonstrated a main effect for intervention $(P=0.002)$, leading to greater BLa values in the BIC group compared with $\mathrm{CHO}$ and ACTIVE, with no change across time. An interaction effect between time and intervention $(P=0.045)$ was found, demonstrating a decrease in BLa for ACTIVE and $\mathrm{CHO}$ compared with stable values in BIC and PASSIVE. Postbout blood $\mathrm{pH}$ values demonstrated main effects for intervention $(P<0.001)$ with no time or interaction effect with higher values in BIC compared with all other interventions. When examining the postbout $\mathrm{HCO}_{3}{ }^{-}$and $\mathrm{BE}$ values there was a main effect for intervention and time (all $P<0.001$ ) with higher values for BIC compared with all other interventions and a decrease in the values from bout to bout. Postbout $\mathrm{PO}_{2}$ values reflected no differences between interventions and no change across sprint bouts. $\mathrm{PCO}_{2}$ was greater in $\mathrm{BIC}$ compared with all other interventions $(P=0.016$ to 0.050$)$ with a reduction from bout 1 to bout $2(P=0.014)$ and bout 1 to bout $3(P=0.006)$.

3.2. Recovery Values after $25 \mathrm{~min}$ Recovery. Relative to the BLa value after the 25 min recovery, there was a main effect for intervention $(P<0.001)$ with no time effect, which demonstrated higher BLa values in PASSIVE compared with all other interventions. In regard to BLa recovery (delta changes from the peak values to the values at the end of the $25 \mathrm{~min}$ recovery phase), a main effect for intervention was found (PASSIVE: $-4.8 \pm 0.3 \mathrm{mmol} / \mathrm{L}$; ACTIVE: $-8.7 \pm 0.4 \mathrm{mmol} / \mathrm{L}$; CHO: $-8.6 \pm 0.2 \mathrm{mmol} / \mathrm{L}$; BIC: $-9.5 \pm 0.3 \mathrm{mmol} / \mathrm{L} ; P<0.001)$ with lowest reduction in BLa in PASSIVE compared with all other interventions and a trend towards greater reduction in BIC compared with $\mathrm{CHO}(P=0.059)$. Furthermore, $\mathrm{CHO}$ had lower recovery compared with BIC $(P=0.037)$. Blood $\mathrm{pH}, \mathrm{HCO}_{3}{ }^{-}$, and $\mathrm{BE}$ values after the $25 \mathrm{~min}$ recovery demonstrated a main effect for intervention (all $P<0.001$ ) with no time or interaction effect with lower values in PASSIVE and higher values in BIC, respectively, compared with all other interventions. There was no difference in $\mathrm{PO}_{2}$ values after $25 \mathrm{~min}$ recovery, while for $\mathrm{PCO}_{2}$ PASSIVE was lower compared with all other interventions $(P=0.010$ to $0.050)$.

3.3. Correlations. Performance in the first bout (test duration) was correlated with peak BLa, peak $\mathrm{pH}$, and the recovery values after $25 \mathrm{~min}$ for $\mathrm{BLa}$ and $\mathrm{pH}$ for PASSIVE ( $r=0.74,-0.88,0.88$, and -0.94 ; all $P<0.001)$, ACTIVE ( $r=0.73,-0.82,0.73$, and -0.69 ; all $P<0.05)$, and $\mathrm{CHO}$ ( $r=0.65,-0.72,0.54$, and -0.63 ; all $P<0.05)$. In the BIC situation only peak $\mathrm{pH}$ and $\mathrm{pH}$ after 25 min recovery were related with performance in the first bout $(r=-0.63, P<$ $0.05 ; r=-0.80, P<0.01)$. No such correlation was found for peak and recovery BLa $(r=0.41,0.53$; both $P>0.05)$.

\section{Discussion}

The main findings of the current study were that (a) the supplementation strategy had no effect on the performance in the first sprint bout, even though marked differences in $\mathrm{pH}, \mathrm{BE}$, and $\mathrm{HCO}_{3}{ }^{-}$were found, particularly in the BIC intervention; (b) repeated sprint bout performance was not affected by supplementation strategy when compared to the placebo active condition (ACTIVE), while placebo with passive rest between the bouts (PASSIVE) resulted in a more pronounced decrease in performance; (c) BIC led to greater $\mathrm{BLa}, \mathrm{pH}, \mathrm{HCO}_{3}{ }^{-}$, and $\mathrm{BE}$ values across the entire experiment compared with all other interventions, while for PASSIVE the opposite was found; (d) BLa values did not change across repeated sprint bouts, though peak $H R$ and performance dropped; (e) though globally not changed, BLa remained constant in BIC and PASSIVE while it decreased in $\mathrm{CHO}$ and ACTIVE across sprint bouts; (f) BLa recovery during the 25 min recovery periods was lowest in PASSIVE compared with all other interventions; recovery in $\mathrm{pH}, \mathrm{BE}$, and $\mathrm{HCO}_{3}{ }^{-}$ was lower in PASSIVE and higher in BIC compared with all 
TABLE 1: Blood lactate, blood gases, $\mathrm{pH}$, and base excess (BE) associated with four interventions at rest, pre-warm-up, post-warm-up, and before and after the three high intensity bouts (mean $\pm \mathrm{SD}$ ).

\begin{tabular}{|c|c|c|c|c|c|c|c|c|c|}
\hline & & Rest & Before warm-up & Warm-up & After bout 1 & Before bout 2 & After bout 2 & Before bout 3 & After bout 3 \\
\hline \multirow{4}{*}{$\begin{array}{l}\text { Blood lactate } \\
(\mathrm{mmol} / \mathrm{L})\end{array}$} & PASSIVE & $1.2 \pm 0.3$ & $1.3 \pm 0.2$ & $3.3 \pm 1.6$ & $14.8 \pm 2.0$ & $9.8 \pm 2.5^{*}$ & $15.2 \pm 2.2$ & $10.5 \pm 2.7^{*}$ & $14.4 \pm 2.5$ \\
\hline & ACTIVE & $1.4 \pm 0.6$ & $1.3 \pm 0.3$ & $3.0 \pm 1.2$ & $14.2 \pm 2.8$ & $5.4 \pm 2.4$ & $13.6 \pm 1.9$ & $5.0 \pm 1.5$ & $13.0 \pm 1.8$ \\
\hline & $\mathrm{CHO}$ & $1.2 \pm 0.2$ & $1.3 \pm 0.2$ & $3.1 \pm 1.4$ & $15.0 \pm 2.0$ & $6.2 \pm 2.2$ & $14.1 \pm 2.3$ & $5.8 \pm 1.8$ & $14.0 \pm 2.0$ \\
\hline & BIC & $1.2 \pm 0.4$ & $1.4 \pm 0.5$ & $3.7 \pm 1.6$ & $15.6 \pm 2.4$ & $6.0 \pm 1.5$ & $15.6 \pm 2.1^{\dagger}$ & $6.3 .5 \pm 1.6^{\ddagger}$ & $15.5 \pm 1.8^{\dagger \neq}$ \\
\hline \multirow{4}{*}{$\mathrm{pH}$} & PASSIVE & $7.41 \pm 0.02$ & $7.41 \pm 0.01$ & $7.41 \pm 0.02$ & $7.16 \pm 0.04$ & $7.29 \pm 0.05^{*}$ & $7.16 \pm 0.04$ & $7.28 \pm 0.06^{*}$ & $7.15 \pm 0.06$ \\
\hline & ACTIVE & $7.43 \pm 0.02$ & $7.43 \pm 0.03$ & $7.41 \pm 0.03$ & $7.18 \pm 0.05$ & $7.39 \pm 0.04$ & $7.19 \pm 0.05$ & $7.39 \pm 0.05$ & $7.19 \pm 0.04$ \\
\hline & $\mathrm{CHO}$ & $7.43 \pm 0.02$ & $7.43 \pm 0.01$ & $7.42 \pm 0.02$ & $7.16 \pm 0.07$ & $7.36 \pm 0.05$ & $7.19 \pm 0.06$ & $7.36 \pm 0.06$ & $7.17 \pm 0.05$ \\
\hline & BIC & $7.41 \pm 0.01$ & $7.47 \pm 0.02^{*}$ & $7.44 \pm 0.01^{\ddagger \$}$ & $7.24 \pm 0.06^{\dagger}$ & $7.43 \pm 0.04^{\dagger}$ & $7.22 \pm 0.06^{*}$ & $7.39 \pm 0.05^{\dagger}$ & $7.22 \pm 0.04^{*}$ \\
\hline \multirow{4}{*}{$\begin{array}{l}\mathrm{HCO}_{3}{ }^{-} \\
(\mathrm{mmol} / \mathrm{L})\end{array}$} & PASSIVE & $24.2 \pm 1.3$ & $24.0 \pm 1.1$ & $21.6 \pm 1.7$ & $12.0 \pm 2.0$ & $13.5 \pm 3.0^{*}$ & $10.1 \pm 1.9^{*}$ & $12.4 \pm 2.0^{*}$ & $9.4 \pm 2.3$ \\
\hline & ACTIVE & $24.2 \pm 1.7$ & $24.4 \pm 1.6$ & $21.9 \pm 1.7$ & $13.0 \pm 2.4$ & $18.1 \pm 3.3$ & $11.4 \pm 2.3$ & $18.4 \pm 2.1$ & $11.8 \pm 1.5$ \\
\hline & $\mathrm{CHO}$ & $24.2 \pm 1.2$ & $24.1 \pm 1.5$ & $21.6 \pm 2.2$ & $12.2 \pm 1.9$ & $17.8 \pm 2.9$ & $11.9 \pm 1.9$ & $18.4 \pm 2.5$ & $11.5 \pm 2.3$ \\
\hline & $\mathrm{BIC}$ & $24.5 \pm 1.4$ & $24.3 \pm 1.2$ & $25.1 \pm 2.7^{*}$ & $15.4 \pm 2.9^{\dagger \$}$ & $21.2 \pm 1.8^{\dagger \$}$ & $13.9 \pm 2.0^{*}$ & $21.1 \pm 2.8^{\dagger \$}$ & $13.5 \pm 2.6^{\$}$ \\
\hline \multirow{4}{*}{$\begin{array}{l}\mathrm{BE} \\
(\mathrm{meq} / \mathrm{L})\end{array}$} & PASSIVE & $-1.7 \pm 1.2$ & $-1.5 \pm 1.2$ & $-4.4 \pm 1.8$ & $-16.8 \pm 2.2$ & $-13.4 \pm 3.6^{*}$ & $-18.6 \pm 2.7^{*}$ & $-14.7 \pm 2.6^{*}$ & $-19.7 \pm 3.1$ \\
\hline & ACTIVE & $-1.6 \pm 1.4$ & $-1.6 \pm 1.5$ & $-4.1 \pm 1.8$ & $-15.5 \pm 3.0$ & $-8.2 \pm 3.8$ & $-17.0 \pm 2.8$ & $-7.7 \pm 2.3$ & $-16.5 \pm 2.0$ \\
\hline & $\mathrm{CHO}$ & $-1.5 \pm 1.3$ & $-1.6 \pm 1.2$ & $-4.3 \pm 2.3$ & $-16.5 \pm 2.4$ & $-8.4 \pm 3.3$ & $-16.6 \pm 2.6$ & $-7.7 \pm 2.8$ & $-17.2 \pm 3.0$ \\
\hline & $\mathrm{BIC}$ & $-1.4 \pm 1.3$ & $-1.1 \pm 1.1$ & $-0.6 \pm 2.7^{*}$ & $-12.6 \pm 3.3^{\dagger \$}$ & $-4.3 \pm 2.2^{\dagger \$}$ & $-13.9 \pm 2.7^{*}$ & $-3.9 \pm 2.1^{\dagger \$}$ & $-14.3 \pm 2.8^{\dagger \$}$ \\
\hline \multirow{4}{*}{$\begin{array}{l}\mathrm{PO}_{2} \\
(\mathrm{mmHg})\end{array}$} & PASSIVE & $78.3 \pm 5.7$ & $78.5 \pm 5.9$ & $82.6 \pm 4.4$ & $93.2 \pm 5.9$ & $83.6 \pm 7.9$ & $95.8 \pm 7.2$ & $84.9 \pm 8.5$ & $98.2 \pm 7.6$ \\
\hline & ACTIVE & $81.4 \pm 9.3$ & $80.1 \pm 8.3$ & $82.0 \pm 4.7$ & $91.7 \pm 9.8$ & $79.4 \pm 3.4$ & $95.9 \pm 4.8$ & $78.5 \pm 5.4$ & $94.7 \pm 6.2$ \\
\hline & $\mathrm{CHO}$ & $83.3 \pm 8.3$ & $81.2 \pm 8.1$ & $84.7 \pm 4.5$ & $94.1 \pm 5.5$ & $81.0 \pm 5.0$ & $93.0 \pm 4.1$ & $77.0 \pm 5.1$ & $93.2 \pm 6.0$ \\
\hline & $\mathrm{BIC}$ & $81.7 \pm 7.5$ & $81.1 \pm 7.7$ & $80.0 \pm 5.9$ & $89.6 \pm 6.1$ & $77.3 \pm 8.2$ & $90.2 \pm 7.0$ & $77.2 \pm 6.9$ & $91.6 \pm 5.0$ \\
\hline \multirow{4}{*}{$\begin{array}{l}\mathrm{PCO}_{2} \\
(\mathrm{mmHg})\end{array}$} & PASSIVE & $38.5 \pm 2.2$ & $38.7 \pm 2.0$ & $35.8 \pm 2.1$ & $34.2 \pm 5.4$ & $28.0 \pm 3.2$ & $28.8 \pm 2.5^{*}$ & $26.7 \pm 2.3^{*}$ & $28.4 \pm 2.6$ \\
\hline & ACTIVE & $37.9 \pm 3.3$ & $37.1 \pm 3.5$ & $36.3 \pm 2.8$ & $35.4 \pm 4.7$ & $32.2 \pm 3.6$ & $30.5 \pm 3.6$ & $32.0 \pm 2.7$ & $31.6 \pm 2.9$ \\
\hline & $\mathrm{CHO}$ & $37.8 \pm 1.9$ & $37.5 \pm 1.1$ & $35.7 \pm 3.4$ & $33.8 \pm 4.9$ & $31.7 \pm 2.6$ & $32.0 \pm 1.8$ & $32.2 \pm 2.7$ & $31.9 \pm 3.1$ \\
\hline & BIC & $39.0 \pm 3.0$ & $39.5 \pm 3.7$ & $38.5 \pm 3.2$ & $37.7 \pm 5.9$ & $33.3 \pm 1.7^{\$}$ & $33.4 \pm 2.9$ & $33.8 \pm 3.0$ & $32.4 \pm 4.2^{\$}$ \\
\hline
\end{tabular}

${ }^{*}$ Different to all other interventions.

${ }^{\dagger}$ Different to CHO.

${ }^{\ddagger}$ Different to ACTIVE.

${ }^{\$}$ Different to PASSIVE.

other interventions; and (g) performance in the first sprint bout was related to higher peak BLa and lower $\mathrm{pH}$ values after exercise and after the $25 \mathrm{~min}$ recovery within all interventions except for BIC where no correlations were found.

4.1. Passive versus Active Recovery. Motivated by the contrasting results of previous research on the effects of active versus passive recovery, the current study supports that active recovery during breaks of longer duration (i.e., $25 \mathrm{~min}$ ) is a favorable strategy compared with passive recovery during repeated high intensity bouts. Active recovery (pedaling at $20 \% \mathrm{VO}_{2 \max }$ ) has been shown to facilitate performance compared to passive recovery in a protocol with 4 times at $110 \%$ of peak performance to exhaustion and $5 \mathrm{~min}$ recovery in between. This was based on an increase in aerobic energy yield and a higher fractional contribution of aerobic metabolism to total energy turnover [16]. Therefore, active recovery may have favored an increased aerobic contribution during the subsequent high intensity bouts and may have promoted an increase in muscle blood flow, $\mathrm{O}_{2}$ delivery, and a correspondent increase in intracellular $\mathrm{pH}$ for all active interventions $[22,23]$. On the contrary, in another study [17] during intermittent exercise of $15 \mathrm{~s}$ high intensity with $15 \mathrm{~s}$ recovery until exhaustion was reached, the passive rest led to increased time to exhaustion compared with active rest using intensity of $40 \% \mathrm{VO}_{2 \max }$ based on lower muscle oxygenation levels during active than during passive recovery. Furthermore, despite comparable responses in acid-base status as in the current study, performance was unaffected by $12 \mathrm{~min}$ active versus passive recovery between 3 high intensity (110\% peak power output) bouts to exhaustion in the study of Siegler et al. [18]. Another explanation for the reduced performance in the PASSIVE situation in the current study might be a decreased metabolic rate and/or priming of the neuromuscular and muscle-tendon system prior to the high intensity bouts.

The low active recovery intensity used in this study was an attempt to increase muscle blood flow while not interrupting $\mathrm{PCr}$ resynthesis, since those stores are crucial for subsequent efforts $[24,25]$. When considering that the half time recovery for $\mathrm{PCr}$ is rarely superior to $90 \mathrm{~s}$ during a passive recovery [26], we expected a more than optimum reload of PCr in the current study for both active and passive interventions. Thus, 


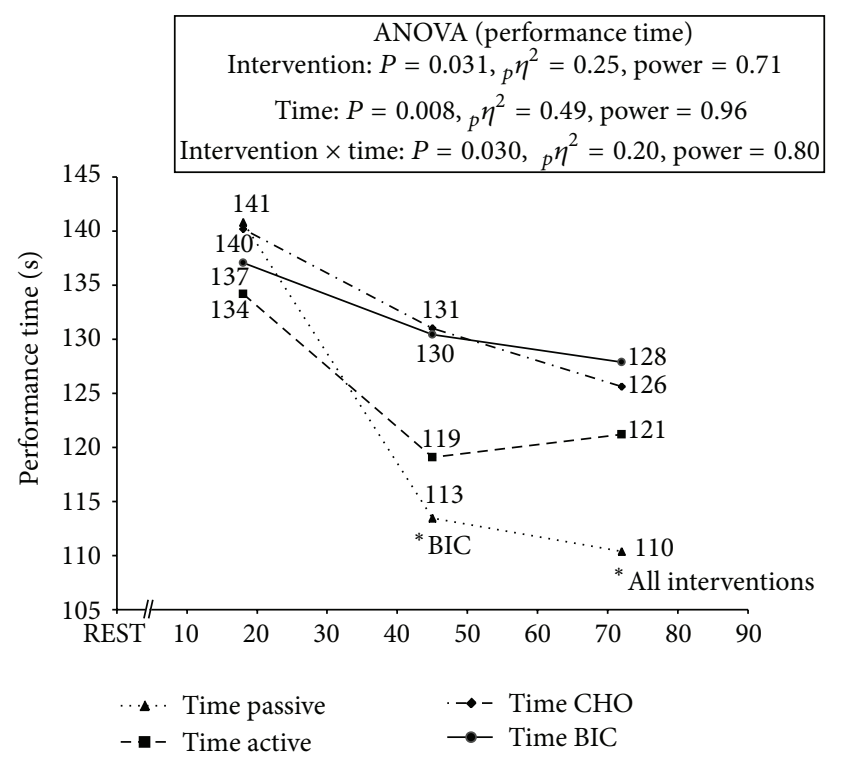

(a)

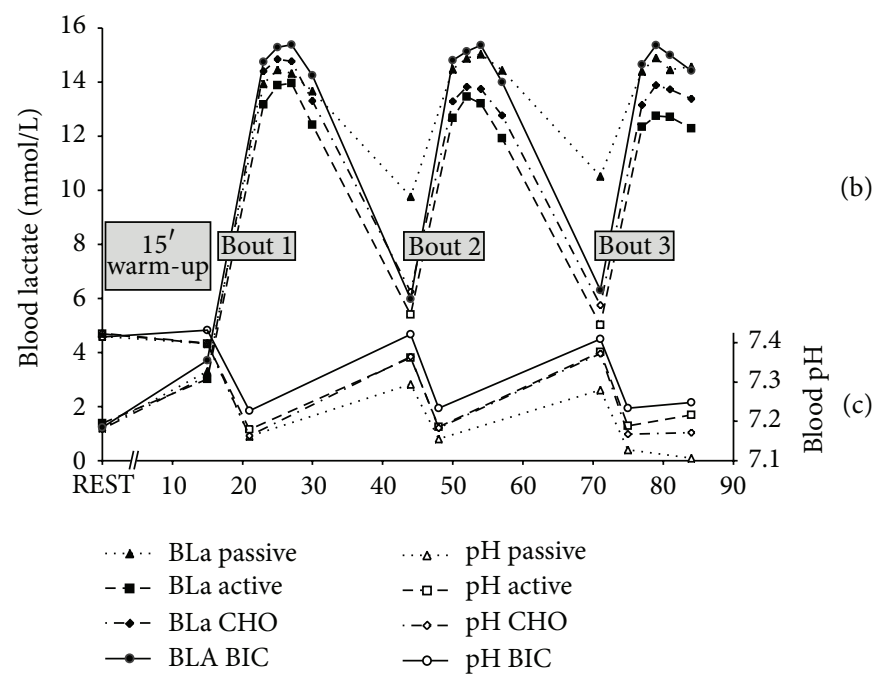

FIGURE 2: Development of running performance (a), blood lactate (b), and $\mathrm{pH}$ (c) across the entire experiment including resting values, postwarm-up values, and postbout values. PASSIVE: passive recovery with placebo; ACTIVE: active recovery with placebo; CHO: active recovery with carbohydrate ingestion; BIC: active recovery with sodium bicarbonate ingestion. ${ }^{*}$ Significant difference to specified intervention.

we may rule out its influence on performance in our bouts without using invasive techniques. Accepting passive recovery to be favored towards PCr resynthesis in short recovery periods between bouts (e.g., up to $2 \mathrm{~min}$ [7]) is probably not imperative when using a much longer $25 \mathrm{~min}$ rest period as in the current study. Therefore, together with the findings of Dorado et al. [16], the current study supports that active recovery seems to lead to superior performance outcome in repeated high intensity bouts when the recovery period is at least $5 \mathrm{~min}$. Further research is warranted concerning the detection of the actual turning point when active recovery outperforms passive recovery and the type of exercise and intensity used during active recovery.

4.2. Effects on Performance. Finding no performance differences between the $\mathrm{CHO}, \mathrm{BIC}$, and placebo intervention (ACTIVE), in contrast to other studies with shorter bout durations and recovery periods (e.g., [5-7, 11, 12]), we conclude that for repeated high intensity performance to exhaustion (bout duration $\sim 2 \mathrm{~min}$ ) with long recoveries $(25 \mathrm{~min}$ ) the length and activity mode of the recovery period may be a more crucial variable than the acid-base status or possibly enhanced glucose availability based on the $\mathrm{CHO}$ supplementation. This idea was previously proposed by Siegler et al. [7]. In order for bicarbonate supplementation to be effective, the metabolic stress must be enough to decrease glycolytic activity and diminish $\mathrm{PCr}$ resynthesis and $\mathrm{Ca}^{2+}$ resequestering in the cell so as to grant a decline in the force production. Furthermore, it would be necessary to maintain this stress situation until the next bout in order to possibly create a significant difference in performance. Though differences in $\mathrm{pH}$ for different active recovery strategies before the second and third bouts were found in the current study, repeated performance was not different between interventions with active recovery.

Concerning $\mathrm{CHO}$ supplementation, neither performance enhancement in the first bout nor increased fatigue resistance across all three bouts can be confirmed. Therefore, in agreement with earlier studies, $\mathrm{CHO}$ supplementation or loading seems to have no effect on sprint performance and/or high intensity exercise up to about 30 min compared with normal diets [27]. To our knowledge, there is only one study demonstrating that a chronic application of carbohydrates over five days resulted in an increase in high intensity performance (e.g., repeated countermovement jump performance with 10 jumps over $60 \mathrm{~s}$ ) compared with a placebo [28]. The current study is in line with the findings of Jenkins et al. $[3,29]$, who demonstrated that there is no improvement in performance while in a carbohydrate loaded state and when ingesting glucose prior to the exercise during five $60 \mathrm{~s}$ allout cycling bouts separated by $5 \mathrm{~min}$ of passive recovery compared with a normal carbohydrate diet. The authors questioned the ergogenic potential of consuming glucose before supramaximal exercise [3]. Also Robinson et al. [30] found no difference between the ingestion of carbohydrates versus placebo during high intensity running (intensity at $\left.100 \% \mathrm{VO}_{2 \max }\right)$ over a duration comparable to that of the current study. Therefore, the speculation of Stöggl et al. [2] that $\mathrm{CHO}$ supplementation might enhance performance in a cross-country skiing sprint competition cannot be supported based on the current findings. However, the finding that peak BLa decreased from bout 1 to bout 2 and that the magnitude of BLa values across the entire simulation was positively related to cross-country skiing sprint performance is in line with the findings of the current study where, in all active rest 
interventions except BIC, BLa decreased from bout 1 to bout 2 and also, in all but the BIC intervention, peak BLa was related to sprint performance. This result might indicate that, especially with BIC, participants show different responses that might have diluted the group outcome. Finally, the ergogenic effect of BLa as proposed by others [31-33] was not noted in this experiment, as PASSIVE and BIC demonstrated a higher prebout BLa but, respectively, lower and similar performance compared with the other interventions.

\section{Limitations}

One limitation of the current study might be seen in the collection of arterialized instead of arterial blood samples for determination of blood gases. In a study of Sauty et al. [34] it was demonstrated that the correlation coefficients between arterial and arterialized blood samples for $\mathrm{PO}_{2}$ and $\mathrm{PCO}_{2}$ were high with 0.928 and 0.957 (both $P<0.001$ ). However, arterialized earlobe $\mathrm{PO}_{2}$ was lower than arterial $\mathrm{PO}_{2}$ in most cases, and the difference increased as arterial $\mathrm{PO}_{2}$ increased. Therefore, the absolute values of $\mathrm{PO}_{2}$ in the current study might underestimate the true $\mathrm{PO}_{2}$ values. However, based on the repeated measured design this fact might not affect the basic outcome.

\section{Conclusion}

In summary, the findings of the current study show that when successive high intensity maximal exhaustion bouts are separated by $25 \mathrm{~min}$ of recovery, an active recovery coupled with a preexercise metabolic alkalosis or carbohydrate supplementation would not lead to detectable improvements in performance as assessed by time to fatigue at high speed treadmill running, regardless of sustaining blood buffering capacity. In spite of a higher $\mathrm{pH}$ than the placebo condition in the case of the bicarbonate supplementation intervention and supposed higher glucose availability in carbohydrates supplementation, we found no significant difference in performance. Only the application of active recovery led to enhanced performance and attenuated fatigue when compared with the passive recovery intervention. Future studies are needed to gain deeper insight into the relationship between exercise intensities and durations with recovery modes and recovery intensities and supplementation after repeated high intensity activity in order to improve performance or delay fatigue.

\section{Conflict of Interests}

The authors declare that there is no personal or financial conflict of interests regarding the publication of this paper.

\section{Acknowledgments}

The authors would like to thank the athletes and research assistant Franz Kreuzhuber for their participation, enthusiasm, and cooperation. Furthermore, the authors would like to express appreciation for the support of Donna Kennedy.

\section{References}

[1] M. Fitzsimons, B. Dawson, D. Ward, and A. Wilkinson, "Cycling and running tests of repeated sprint ability," Australian Journal of Science and Medicine in Sport, vol. 25, no. 4, pp. 82-87, 1993.

[2] T. Stöggl, S. Lindinger, and E. Müller, "Analysis of a simulated sprint competition in classical cross country skiing," Scandinavian Journal of Medicine \& Science in Sports, vol. 17, no. 4, pp. 362-372, 2007.

[3] D. G. Jenkins, C. A. Hutchins, and D. Spillman, “The influence of dietary carbohydrate and pre-exercise glucose consumption on supramaximal intermittent exercise performance," British Journal of Sports Medicine, vol. 28, no. 3, pp. 171-176, 1994.

[4] M. Vogt, A. Puntschart, M. Angermann et al., "Metabolic consequences of a simulated slalom competition training of recreational alpine skiers," Leistungssport, vol. 35 , no. 2, pp. $48-$ 54, 2005.

[5] D. Bishop and B. Claudius, "Effects of induced metabolic alkalosis on prolonged intermittent-sprint performance," Medicine and Science in Sports and Exercise, vol. 37, no. 5, pp. 759-767, 2005.

[6] D. L. Costill, F. Verstappen, H. Kuipers, E. Janssen, and W. Fink, "Acid-base balance during repeated bouts of exercise: influence of $\mathrm{HCO}_{3}$," International Journal of Sports Medicine, vol. 5, no. 5, pp. 228-231, 1984.

[7] J. C. Siegler, L. R. McNaughton, A. W. Midgley, S. Keatley, and A. Hillman, "Metabolic alkalosis, recovery and sprint performance," International Journal of Sports Medicine, vol. 31, no. 11, pp. 797-802, 2010.

[8] P. Krustrup, M. Mohr, A. Steensberg, J. Bencke, M. Klær, and J. Bangsbo, "Muscle and blood metabolites during a soccer game: Implications for sprint performance," Medicine and Science in Sports and Exercise, vol. 38, no. 6, pp. 1165-1174, 2006.

[9] J. B. Leiper, A. S. Prentice, C. Wrightson, and R. J. Maughan, "Gastric emptying of a carbohydrate-electrolyte drink during a soccer match," Medicine and Science in Sports and Exercise, vol. 33, no. 11, pp. 1932-1938, 2001.

[10] C. R. Correia-Oliveira, R. Bertuzzi, M. A. P. Dal'Molin Kiss, and A. E. Lima-Silva, "Strategies of dietary carbohydrate manipulation and their effects on performance in cycling time trials," Sports Medicine, vol. 43, no. 8, pp. 707-719, 2013.

[11] L. M. Burke and D. B. Pyne, "Bicarbonate loading to enhance training and competitive performance," International Journal of Sports Physiology and Performance, vol. 2, no. 1, pp. 93-97, 2007.

[12] D. Bishop, J. Edge, C. Davis, and C. Goodman, "Induced metabolic alkalosis affects muscle metabolism and repeatedsprint ability," Medicine and Science in Sports and Exercise, vol. 36, no. 5, pp. 807-813, 2004.

[13] A. J. Carr, W. G. Hopkins, and C. J. Gore, "Effects of acute alkalosis and acidosis on performance: a meta-analysis," Sports Medicine, vol. 41, no. 10, pp. 801-814, 2011.

[14] G. Lavender and S. R. Bird, "Effect of sodium bicarbonate ingestion upon repeated sprints," British Journal of Sports Medicine, vol. 23, no. 1, pp. 41-45, 1989.

[15] D. Bishop, J. Edge, and C. Goodman, "Muscle buffer capacity and aerobic fitness are associated with repeated-sprint ability in women," European Journal of Applied Physiology, vol. 92, no. 45, pp. 540-547, 2004.

[16] C. Dorado, J. Sanchis-Moysi, and J. A. Calbet, "Effects of recovery mode on performance, $\mathrm{O}_{2}$ uptake, and $\mathrm{O}_{2}$ deficit during high-intensity intermittent exercise," Canadian Journal of Applied Physiology, vol. 29, no. 3, pp. 227-244, 2004. 
[17] G. Dupont, W. Moalla, C. Guinhouya, S. Ahmaidi, and S. Berthoin, "Passive versus active recovery during high-intensity intermittent exercises," Medicine \& Science in Sports \& Exercise, vol. 36, no. 2, pp. 302-308, 2004.

[18] J. C. Siegler, J. Bell-Wilson, C. Mermier, E. Faria, and R. A. Robergs, "Active and passive recovery and acid-base kinetics following multiple bouts of intense exercise to exhaustion," International Journal of Sport Nutrition and Exercise Metabolism, vol. 16, no. 1, pp. 92-107, 2006.

[19] L. R. McNaughton, "Bicarbonate ingestion: effects of dosage on 60 s cycle ergometry," Journal of Sports Sciences, vol. 10, no. 5, pp. 415-423, 1992.

[20] K. Tsintzas, C. Williams, D. Constantin-Teodosiu et al., "Phosphocreatine degradation in type I and type II muscle fibres during submaximal exercise in man: effect of carbohydrate ingestion," Journal of Physiology, vol. 537, part 1, pp. 305-311, 2001.

[21] K. Currell and A. E. Jeukendrup, "Validity, reliability and sensitivity of measures of sporting performance," Sports Medicine, vol. 38, no. 4, pp. 297-316, 2008.

[22] N. J. Berger, L. R. McNaughton, S. Keatley, D. P. Wilkerson, and A. M. Jones, "Sodium bicarbonate ingestion alters the slow but not the fast phase of VO2 kinetics," Medicine \& Science in Sports \& Exercise, vol. 38, no. 11, pp. 1909-1917, 2006.

[23] S. C. Forbes, G. H. Raymer, J. M. Kowalchuk, and G. D. Marsh, " $\mathrm{NaHCO}_{3}$-induced alkalosis reduces the phosphocreatine slow component during heavy-intensity forearm exercise," Journal of Applied Physiology, vol. 99, no. 5, pp. 1668-1675, 2005.

[24] L. Nybo, O. Girard, M. Mohr, W. Knez, S. Voss, and S. Racinais, "Markers of muscle damage and performance recovery after exercise in the heat," Medicine and Science in Sports and Exercise, vol. 45, no. 5, pp. 860-868, 2013.

[25] A. Vanhatalo, L. R. McNaughton, J. Siegler, and A. M. Jones, "Effect of induced alkalosis on the power-duration relationship of "all-out" exercise," Medicine \& Science in Sports \& Exercise, vol. 42, no. 3, pp. 563-570, 2010.

[26] B. Dawson, C. Goodman, S. Lawrence et al., "Muscle phosphocreatine repletion following single and repeated short sprint efforts," Scandinavian Journal of Medicine and Science in Sports, vol. 7, no. 4, pp. 206-213, 1997.

[27] A. Jeukendrup and M. Gleeson, Sport Nutrition, Human Kinetics, Leeds, UK, 2010.

[28] C. A. Koenig, D. Benardot, M. Cody, and W. R. Thompson, "Comparison of creatine monohydrate and carbohydrate supplementation on repeated jump height performance," Journal of Strength and Conditioning Research, vol. 22, no. 4, pp. 1081-1086, 2008.

[29] D. G. Jenkins, J. Palmer, and D. Spillman, "The influence of dietary carbohydrate on performance of supramaximal intermittent exercise," European Journal of Applied Physiology and Occupational Physiology, vol. 67, no. 4, pp. 309-314, 1993.

[30] E. M. Robinson, L. B. Graham, J. Moncada, B. Jensen, M. Jones, and S. A. Headley, "Carbohydrate-electrolyte ingestion has no effect on high intensity running performance or blood metabolites," Journal of Exercise Physiology Online, vol. 5, no. 1, pp. 49-55, 2002.

[31] G. A. Brooks, "Lactate doesn't necessarily cause fatigue: why are we surprised?" The Journal of Physiology, vol. 536, no. 1, p. 1, 2001.

[32] L. B. Gladden, "Lactate metabolism: a new paradigm for the third millennium," Journal of Physiology, vol. 558, part 1, pp. 530, 2004.
[33] B. F. Miller, J. A. Fattor, K. A. Jacobs et al., "Lactate and glucose interactions during rest and exercise in men: effect of exogenous lactate infusion," Journal of Physiology, vol. 544, no. 3, pp. 963975, 2002.

[34] A. Sauty, C. Uldry, L.-F. Debétaz, P. Leuenberger, and J.-W. Fitting, "Differences in $\mathrm{PO}_{2}$ and $\mathrm{PCO}_{2}$ between arterial and arterialized earlobe samples," European Respiratory Journal, vol. 9, no. 2, pp. 186-189, 1996. 

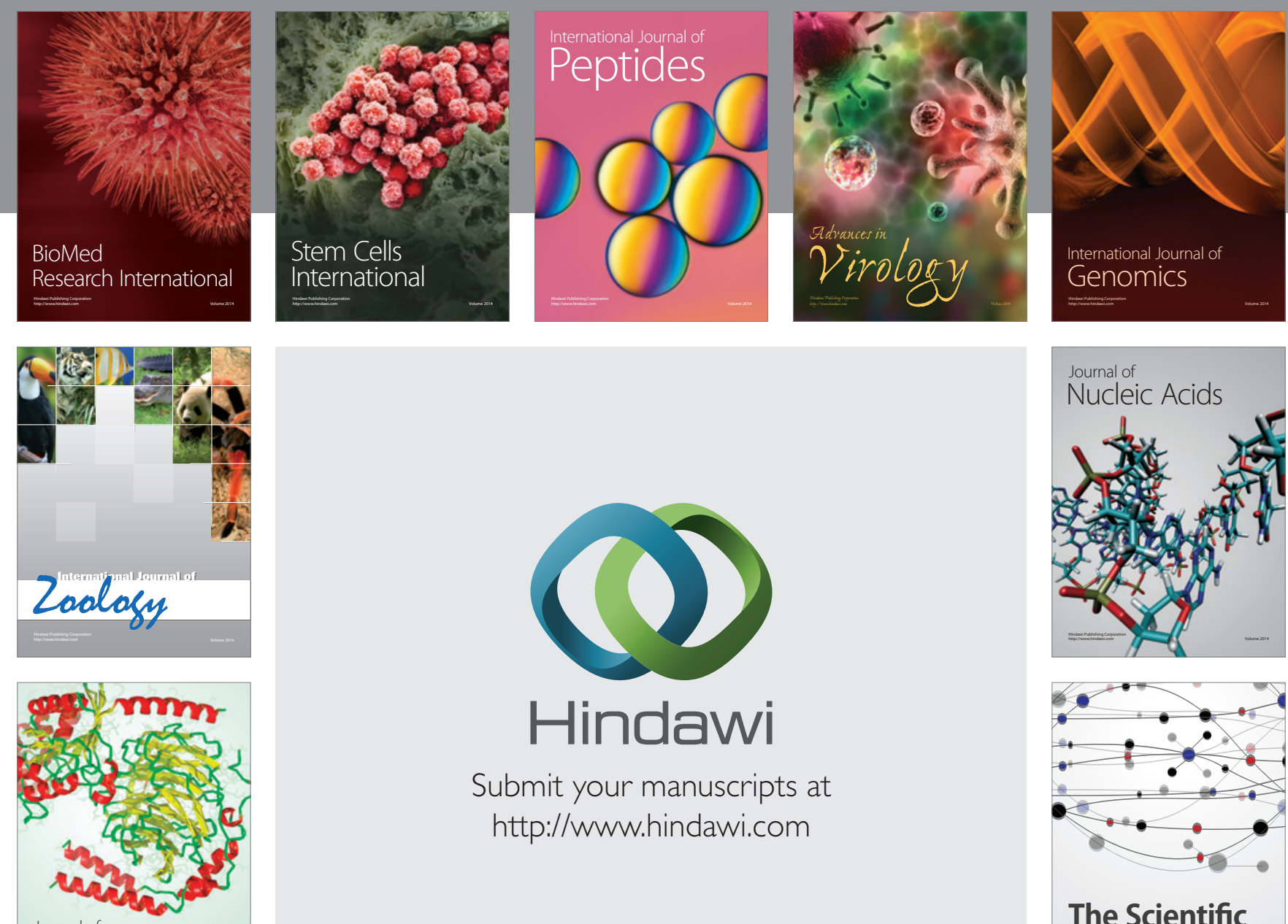

Submit your manuscripts at

http://www.hindawi.com

Journal of
Signal Transduction
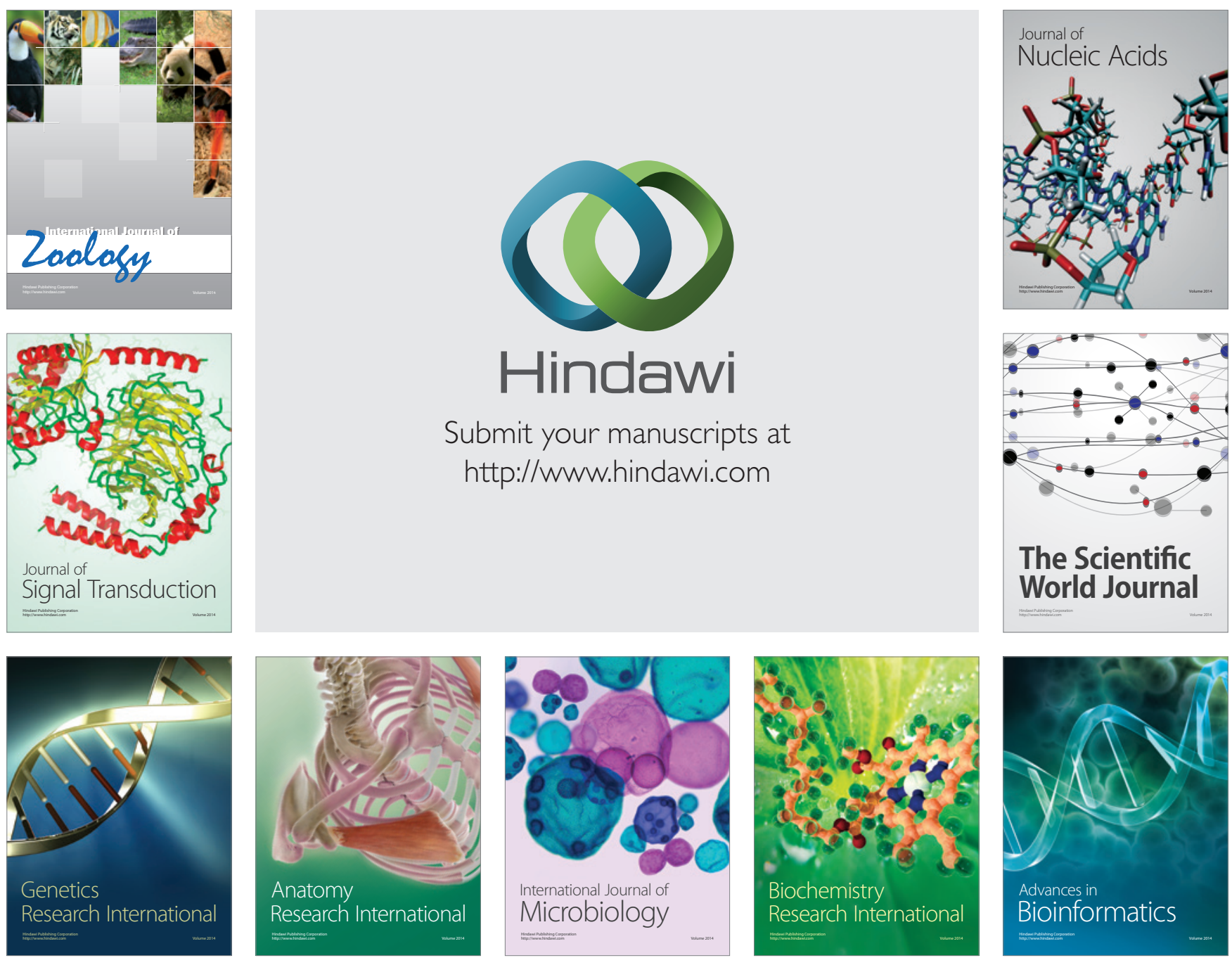

The Scientific World Journal
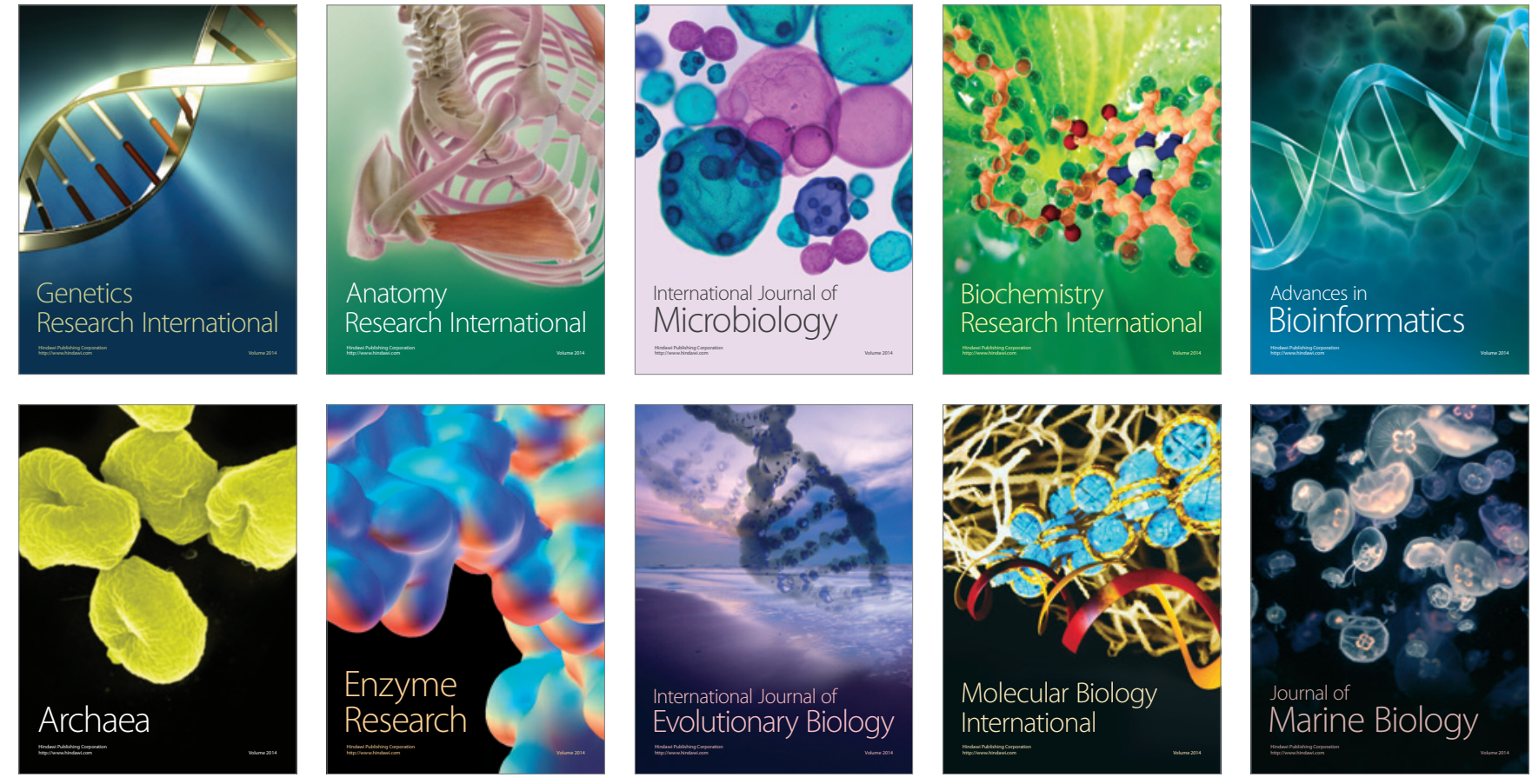